\title{
Strokes In Young Adults And Children
}

Farhad Iranmanesh, M D,.

Professor of Neurology, Stroke fellowship, Kerman Medical University, Kerman, Iran.

Stroke is in second place on a mortality list in the world. Also, stroke is a leading cause of disability. Approximately $20 \%$ of all strokes occur in Children and young adults. The etiology of stroke in Children and young adults is different from that in older patients, and has an influence on diagnostic evaluation and treatment, so knowledge about older patients cannot always be applied to these patients. The list of stroke etiologies among young adults and children is extensive. Ischemic stroke are more frequent than hemorrhagic strokes in both groups. Stroke in young adults had been thought to be associated with risk factors, including arterial (such as dissection, reversible cerebral vasoconstriction syndrome, inflammatory arteritis ,moyamoya ,migraine - induced stroke, genetic or inherted arteriopathy, premature atherosclerosis ) cardiac (such as patent foramen ovale, cardiomyopathy , congenital heart disease) and hematologic (such as deficiencies of protein S,protein C,or antithrombin;factor V lieden mutation). Common risk factors for stroke in children include: Sickle-cell disease, diseases of the arteries, abnormal blood clotting, head or neck trauma. There are no specific recommendations or guidelines for primary or secondary stroke prevention in young adults. Primary prevention focused on identifying and managing known vascular risk factors, such as hypertension, disorders of lipid metabolism, and diabetes, and non-drug strategies and lifestyle changes, including smoking, reducing body weight, increasing regular aerobic physical activity, and adopting a healthy diet with more fruit and vegetables and less salt. For secondary stroke prevention, identification of the etiologic mechanism of the initial stroke and the presence of any additional risk factors is most important. It consists of optimal treatment of vascular risk factors administering antiplatelet or anticoagulant therapy, and if indicated, invasive surgical or endovascular therapeutic procedures. An integral part of this is lifestyle changes, emphasizing regular physical activity, a diet low in salt and saturated fat and high in fruit and vegetables, reducing overweight and quitting smoking.

Key words: stroke, disability

DOI: $10.7575 /$ aiac.abcmed.ca1.55

A I Published Date: February 2017 